\title{
涡流发生器对动态失速影响的模拟研究*
}

赵振宙 ${ }^{1,2}$ 苏德程 $^{1}$ 王同光 $^{3}$ 吴 吴 $^{1}$ 孟令玉 $^{1}$ 许波峰 ${ }^{1}$ 郑 源 $^{1}$

(1. 河海大学能源与电气学院 南京 211100;

2. 内蒙古工业大学风能太阳能利用技术教育部重点实验室 呼和浩特 010000 ;

3. 南京航空航天大学江苏省风力机设计高技术研究重点实验室 南京 210016)

\begin{abstract}
摘要: 基于风力机专用翼型 DU91-W2-250 直叶片段, 采用延迟分离浴模拟(Delayed detached-eddy simulation, DDES)与 $k-\omega$ SST 两种 CFD 模拟方法, 研究了涡流发生器(Vortex generators, VGs)对动态失速的影响, 并对两种模拟方法的表现进行了比较。 结果表明: 动态失速下, 光滑叶片段的分离与重新附着均出现延迟; 加 VGs 后, 上仰阶段叶片段的气动分离更加延迟, 下俯 阶段叶片段上表面附着流动重建的更早, 气动力的迟滞现象得到明显改善。VGs 对 DU91-W2-250 叶片段增升减阻的效果明 显, 其中最大升力系数增加 $10 \%$, 周期平均升力系数增加 $17.9 \%$, 最大阻力系数减少 $56.3 \%$, 周期平均阻力系数减少 $40.3 \%$ 。 DDES 模型能更细致地反映 VGs 对动态失速抑制作用下叶片段表面复杂流动分离现象, $k-\omega$ SST 模型则难以捕捉小尺度浴结 构, 模拟得到的涡结构展向呈二维分布。
\end{abstract}

关键词: 动态失速; 涡流发生器; DDES 方法; DU91-W2-250

中图分类号: TK81

\section{Simulation Study on the Effect of Vortex Generators on Dynamic Stall}

\author{
ZHAO Zhenzhou $^{1,2}$ SU Decheng $^{1} \quad$ WANG Tongguang $^{3} \quad$ WU Hao $^{1}$ \\ MENG Lingyu $^{1}$ XU Bofeng ${ }^{1}$ ZHENG Yuan ${ }^{1}$ \\ (1. College of Energy and Electrical Engineering, Hohai University, Nanjing 211100; \\ 2. Key laboratory of wind Energy and Solar Energy Technology, \\ Inner Mongolia University of Technology, Hohhot 010000; \\ 3. Jiangsu Key Laboratory of Hi-Tech Research for Wind Turbine Design, \\ Nanjing University of Aeronautics \& Astronautics, Nanjing 210016)
}

\begin{abstract}
The effects of vortex generators (VGs) on dynamic stall are investigated based on a blade segment using the airfoil of DU91-W2-250. The investigation is numerically performed by two simulation approaches, i.e. Delayed detached-eddy simulation (DDES) and $k$ - $\omega$ SST, and their results are compared. The results show that of airflow separation and reattachment within the dynamic stall process are delayed on the smooth blade surface. After adding VGs, the airflow separation is delayed at the pitch up stage, the reattachment of air flow on the up surface becomes earlier when the blade is pitching down, and the aerodynamic hysteresis is obviously improved. The magnitudes of lift increased and the drag reduced by VGs of are substantial. In detail, the maximum lift coefficient of DU91-W2-250 blade segment is increased by $10 \%$, the average value is increased by $17.9 \%$; the maximum drag coefficient is decreased by $56.3 \%$, and the average value is decreased by $40.3 \%$. DDES model can demonstrate clearly the complex flow separation occurred in dynamic stall as it is under the effect of VGs, while the $k$ - $\omega$ SST model hardly provides a deep insight into the small-scale vortices structure and which furthermore shows two-dimensional characteristics.
\end{abstract}

Key words: dynamic stall; vortex generator; DDES method; DU91-W2-250

\section{0 前言}

风力机叶片根部厚翼型在大攻角下易发生气流

* 风能太阳能利用技术教育部重点实验室开放基金(20182D05)和国家自 然科学基金 (51876054, 11502070)资助项目。20190409 收到初稿, 20190928 收到修改稿
分离现象, 这对叶片的气动性能产生不利影响。研 究和实践均表明, 浴流发生器(Vortex generators, $\mathrm{VGs}$ )安装在叶片上翼面, 可延迟边界层的气流分 离, 有效改善叶片气动特性 ${ }^{[1-2]}$ 。不少学者, 通过试 验和 CFD 方法对 VGs 延迟气动分离的机理进行了 研究。典型的有: 文献[3-4]对加 VGs 的 DU 系列 
钝尾缘厚翼型的气动性能进行了风洞试验, 试验结 果表明 VGs 能够增大翼型失速攻角和最大升阻比。 ZHANG 等 ${ }^{[5]}$ 对 DU91-W2-250 风力机专用翼型进行 了 $\mathrm{CFD}$ 模拟, 验证了 VGs 改变上翼面边界层的逆 压梯度分布的有效性, 揭示了 VGs 控制表面流动的 作用机理。MONOLESOS 等 ${ }^{[6]}$ 采用 PIV 和油膜试验, 在雷诺数 $0.87 \times 10^{6}$ 工况下对二维叶片表面 VGs 下游 流动拓扑结构进行了可视化试验研究, 给出了 VGs 作用下叶片表面流态分布和 VGs 脱落浴结构。叶片 流动控制整体效果受到了 VGs 几何尺寸、安装位 置、布局方式等特征因素的影响。为此, 不少学者 针对不同的翼型进行研究, 并给出了 VGs 结构特 征因素对叶片分离浴的控制作用规律, 总的来讲, 三角翼形状比矩形翼更有效, 反向旋转布置比同 向旋转布置有效, $\mathrm{VGs}$ 为边界层厚度 $20 \% \sim 50 \%$ 时可有效减小叶片表面阻力但抑制效果不发生改 变等 ${ }^{[7-10]}$ 。这些文献集中研究了 VGs 对翼型静态失 速的作用规律，提高了 VGs 对风力机叶片表面流 动控制机理的认知水平。然而, 在实际风况中, 偏 航、变桨、剪切风、地面边界层效应等影响下, 叶 片在更多的时间内因攻角随时间发生连续变化而 产生动态失速现象 ${ }^{[11-14]}$ 。从光滑叶片研究可知, 动 态失速工况下, 叶片表面的分离流动更加复杂, 失 速攻角远比静态条件下的失速攻角要大, 气动力特 性随攻角变化的曲线出现明显的迟滞现象, 且具有 复杂的波动性 ${ }^{[15-16]}$ 。那么, VGs 对风力机叶片动态 失速具有怎样的影响, 现有文献研究还极少。仅有 文献 ${ }^{[17]}$ 采用基于 RANS 方法的 $k-\omega$ SST 模型对此 问题进行了初步研究, 结果表明 VGs 能够显著提 高翼型动态失速下的气动性能。然而, VGs 对动态 失速气动性能影响的研究与认识还远远不够, 有待 进一步研究。

分离浴模拟(Detached-eddy simulation, DDES) 是 RANS 方法和大涡模拟(Large eddy simulation, LES)方法的混合算法, 很好地结合了 RANS 方法在 边界层处理优势和 LES 方法对浴结构模拟精度高、 适用性好的优点 ${ }^{[18]}$ 。较 RANS 方法而言, DES 更加 适合模拟复杂的流动分离现象, 可以更精细地捕捉 叶片分离浴结构 ${ }^{[19-20]}$ 。文献[21]为解决 DES 方法的 模型应力损失问题, 提出了改良后的延迟分离浴模 拟(DDES)方法。LETTGUS 等 ${ }^{[22]}$ 发现 DDES 方法能 更加细致地描述动态失速下的流动分离现象。

为此, 采用 DDES 和 $k-\omega \mathrm{SST}$ 两种模拟方法, 研究 VGs 对风力机翼型 DU91-W2-250 动态失速下 气动性能的影响规律, 并针对 DDES 模拟方法的表 现进行讨论。

\section{1 数值模拟方法}

\section{1 模拟方法}

流体流动采用 Navier-Stokes 方程模拟，其雷诺 平均控制方程为

$$
\begin{gathered}
\frac{\partial}{\partial x_{i}}\left(\bar{u}_{i}\right)=0 \\
\frac{\partial}{\partial t}\left(\rho \bar{u}_{i}\right)+\frac{\partial}{\partial x_{j}}\left(\rho \bar{u}_{i} \bar{u}_{j}\right)=-\frac{\partial \bar{p}}{\partial x_{i}}+ \\
\frac{\partial}{\partial x_{j}}\left[\mu\left(\frac{\partial \bar{u}_{i}}{\partial x_{j}}+\frac{\partial \bar{u}_{j}}{\partial x_{i}}\right)\right]+\frac{\partial}{\partial x_{j}}\left(-\rho \bar{u}_{i} \bar{u}_{j}\right)
\end{gathered}
$$

$k-\omega \mathrm{SST}$ 模型的输运方程为

$$
\begin{gathered}
\frac{\mathrm{D} k}{\mathrm{D} t}=P_{k}-\beta^{*} \omega k+\frac{\partial}{\partial x_{j}}\left(\left(v+\sigma_{k} v_{t}\right) \frac{\partial k}{\partial x_{j}}\right) \\
\frac{\mathrm{D} \omega}{\mathrm{D} t}=\gamma \frac{\omega}{k} P_{k}-\beta \omega^{2}+\frac{\partial}{\partial x_{j}}\left(\left(v+\sigma_{\omega} v_{t}\right) \frac{\partial \omega}{\partial x_{j}}\right)+ \\
2\left(1-F_{1}\right) \sigma_{\omega 2} \frac{1}{\omega} \frac{\partial k}{\partial x_{j}} \frac{\partial \omega}{\partial x_{j}}
\end{gathered}
$$

式中, $k$ 为湍动能, $\omega$ 为比耗散率, $P_{k}$ 是湍流生成 率, $v_{t}$ 为浴黏系数, $v$ 为湍动黏度, $\beta^{*}$ 为常数参数, $\gamma 、 \sigma_{k} 、 \beta 、 \sigma_{\omega} 、 F_{1}$ 为变量参数。

DES 方法在近壁边界层内使用 RANS 方法, 避 免了 LES 方法近壁面网格划分难的不足, 在远离壁 面主流区采用 LES 方法而可以更精细地捕捉涡结 构 ${ }^{[23]}$ 。本文中 DDES 模型在近壁区采用 $k-\omega \mathrm{SST}$ 模 型。由于篇幅原因，本文 DDES 模型的具体内容不 再阐述，详见文献[18]。

\section{2 几何模型与网格}

DU91 系列翼型是荷兰 Delft 大学设计的风力机 专用翼型, 其中 DU91-W2-250 最大厚度为 $25 \%$ 弦 长, 位于距前缘点 $32.2 \%$ 弦长。因很多文献对其气 动载荷试验数据进行记载, 可供参考, 本文将基于 该翼型展开研究。DU91-W2-250 直叶片段展长 0.105 $\mathrm{m}$ 、弦长为 $0.6 \mathrm{~m}$, 在 $20 \%$ 弦长处布置三组反向旋转 排列的 VGs。VGs 为三角翼结构, 弦长 $17 \mathrm{~mm}$, 高 $5 \mathrm{~mm}$, 攻角为 $16.4^{\circ}$, 组间距 $35 \mathrm{~mm}$, VGs 结构尺 寸示意如图 1 所示。

采用 ICEM 软件来划分结构化网格, 模型计算 域为圆形计算域。计算域分为静止的外域与运动的 内域, 外域半径为 20 倍弦长, 内域半径为 3 倍弦长, 如图 $2 \mathrm{a}$ 。翼面第一层网格满足条件 $y^{+}<1$, 边界层处 网格法向增长率为 1.1 。三角翼 VGs 采用无厚度三 
角形面网格代替, 此时若设无厚度三角形面为内部 边界时, 几何模型转变为光滑叶片段; 若设这些三 角形面为无滑移壁面条件时, 几何模型即为加 VGs 的叶片段。这样处理的优势为光滑叶片段和加 VGs 叶片段的网格划分相同, 其计算结果更具有对比性。 叶片段处网格结构如图 $2 \mathrm{~b}$, 网格总数为 500 万。

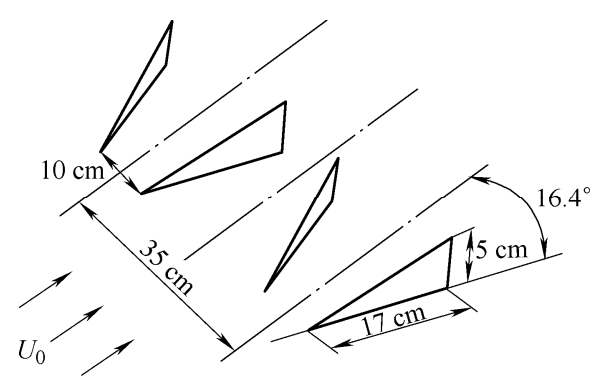

图 1 VGs 尺寸结构

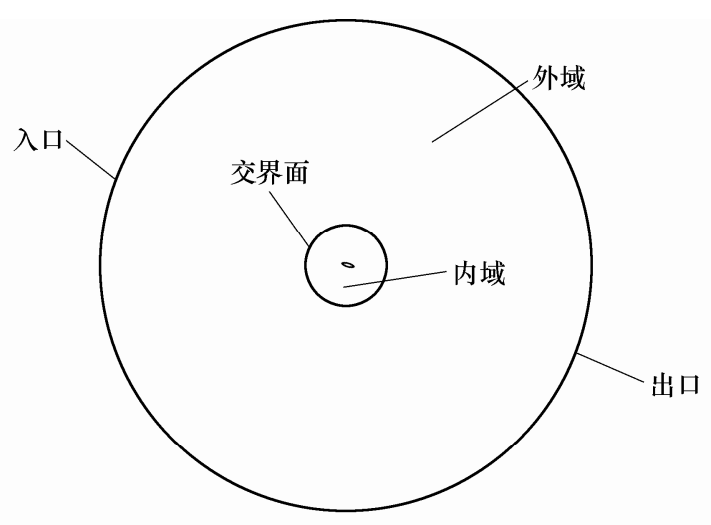

(a) 计算域

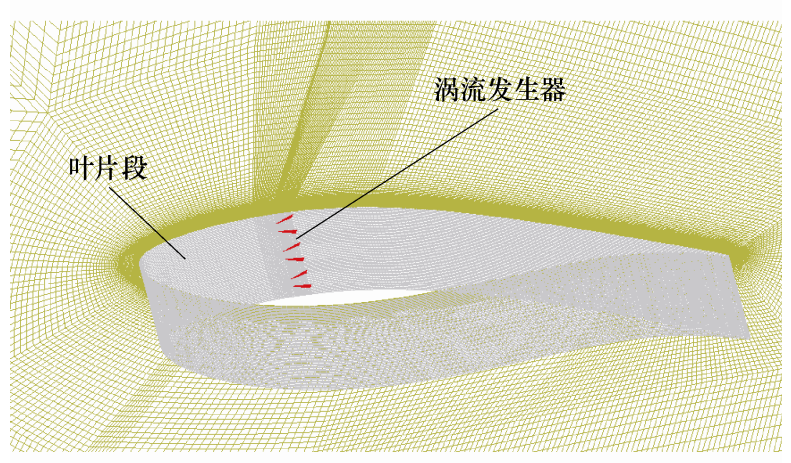

(b) 叶片段处网格结构

图 2 计算模型

\section{3 计算条件}

计算雷诺数为 $2 \times 10^{6}$, 空气密度 $\rho=1.225 \mathrm{~kg} / \mathrm{m}^{3}$, 动力黏度 $\mu=1.7894 \times 10^{-5} \mathrm{~kg} /(\mathrm{m} \cdot \mathrm{s})$ 。采用速度入口 边界条件, 速度大小为 $48.69 \mathrm{~m} / \mathrm{s}$, 入口湍流度为 $0.03 \%$, 出口为压力出口条件, 叶片段表面为无滑 移壁面条件。外域与内域的交界面设定为 interface 条件并处理成交界面对, 两侧面为对称边界条件。 采用 UDF 实现内域和叶片段的俯仰振荡运动, 设定 数值格式均为二阶, 时间步长为 $0.001 \mathrm{~s}$ 。

将动态失速过程视为叶片段以正弦规律进行的
俯仰振荡运动。攻角变化规律为

$$
\alpha=\alpha_{0}+\Delta \alpha \cos \left(\frac{2 k U}{c} t\right)
$$

式中, 初始攻角 $\alpha_{0}=14^{\circ}$, 振幅 $\Delta \alpha=10^{\circ}$, 折合频 率 $k$ 取为 $0.078, U$ 为来流速度, $c$ 为弦长, $t$ 为时 间, 取第四个计算周期的结果进行分析。

\section{2 计算结果与分析}

\section{1 模拟方法精度验证}

为验证数值方法的可靠性, 将加 VGs 的 DU91-W2-250 叶片段静态失速过程, 分别采用 DDES 和 $k-\omega$ SST 模拟方法进行预测, 计算值与文 献[3]的试验值进行对比, 结果如图 3 所示。从图可 看出, VGs 使 DU91-W2-250 静态失速攻角从 $10^{\circ}$ 延迟到 $17^{\circ}$ 左右, 最大升力系数由 1.4 增大至近 2.0 。 对比两模拟方法计算结果发现, 在 $\alpha<15^{\circ}$ 范围内试 验值与两方法模拟结果吻合均良好; 在 $\alpha>15^{\circ}$, 加 VGs 叶片表面逐渐出现分离, 此时 DDES 模拟值与 试验值仍然吻合良好; $k-\omega \mathrm{SST}$ 模型未能准确地预 测到叶片分离过程, 导致计算结果与试验值偏差较 大, 需要更加精细的网格才能减小误差, 这无疑增 加了工作量。总体而言, DDES 模型模拟对叶片段 失速后气动性能的预测表现更好。

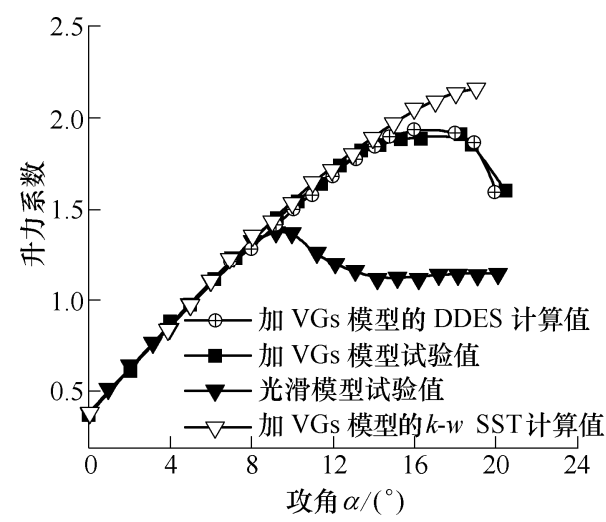

图 3 试验值与模型计算值对比

\subsection{DDES 模型下动态失速 CFD 模拟分析}

\subsection{1 光滑叶片段的动态失速分析}

图 4 和图 5 分别为光滑叶片段在一个周期内升、 阻力系数随攻角变化的 DDES 计算结果。在动态失 速条件下, 升阻力系数曲线呈迟滞环状, 气动力迟 滞现象严重。DU91-W2-250 叶片段在 $10^{\circ}$ 左右静态 失速, 最大升力系数为 1.37 ; 从图 4、5 可以看出, 动态失速下叶片段出现明显的失速延迟, 失速攻角 延迟至 $21^{\circ}$, 最大升力系数达 2.17 , 分离点(图 4 中 $B$ 点)后升阻力系数有波动现象, 相比静态载荷, 叶 片段承受的动态载荷更加复杂严峻。 


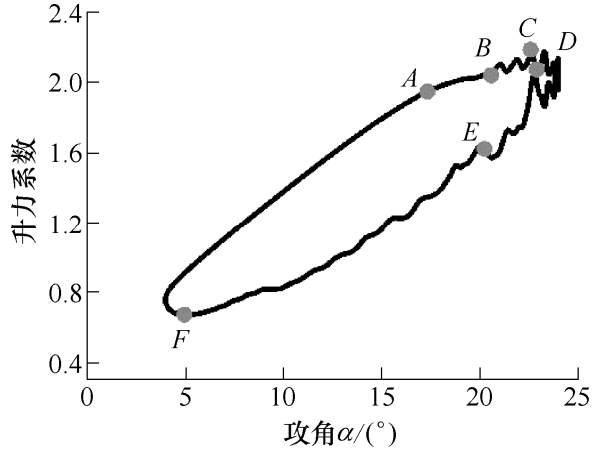

图 4 光滑叶片段升力系数迟滞曲线

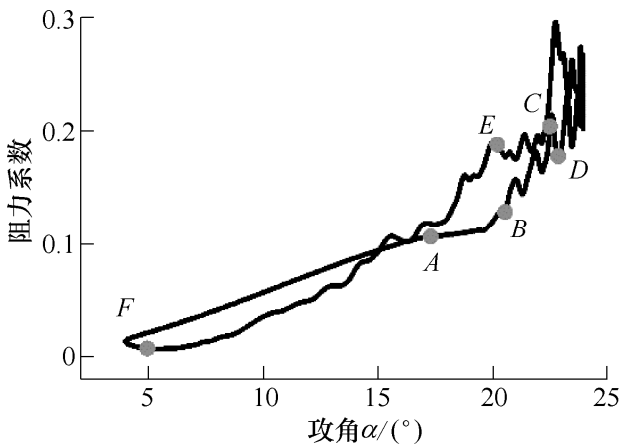

图 5 光滑叶片段阻力系数迟滞曲线

图 6a～6f 分别为图 4 和图 5 中失速过程中 $A 、 B 、$ $C 、 D 、 E 、 F$ 点对应的叶片段展向中心剖面流线图。 结合不同时刻的流动拓扑结构分布, 来解释上述升、 阻力系数的变化趋势。如图 6 显示, 在叶片段上仰 时, 在 $\alpha<17.34^{\circ}(A$ 点之前 $)$, 光滑叶片段的升阻力系 数随攻角线性增大; 当上仰到 $A$ 点时, 光滑叶片段 后缘处的边界层开始出现分离迹象, 升力系数增长 变缓。当光滑叶片段上仰至 $\alpha=20.60^{\circ}$ (B 点)时, 上 翼面后缘有明显的不同尺度的浴形成, 导致 $B$ 点后 光滑叶片段的升阻力系数开始不断波动, 如 $C-D$ (图 4、5)过程。光滑叶片段上仰至 $C$ 点(图 4、5)时, 攻 角为 $22.54^{\circ}$, 升力系数达到最大值 2.18 ; 当叶片段

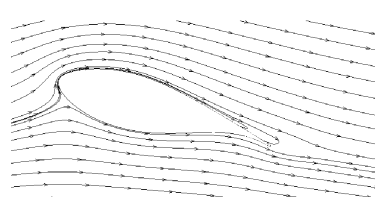

(a) $\alpha=17.34^{\circ}$ ( 上仰 )

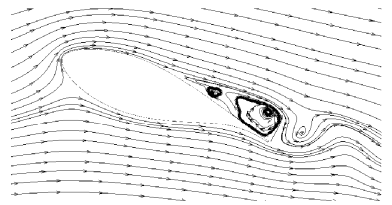

(c) $\alpha=22.54^{\circ}$ ( 上仰 )

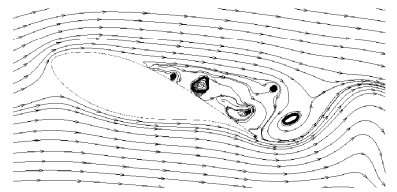

(e) $\alpha=22.25^{\circ}$ (下俯 )

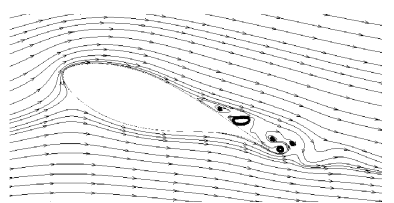

(b) $\alpha=20.60^{\circ}$ ( 上仰 )

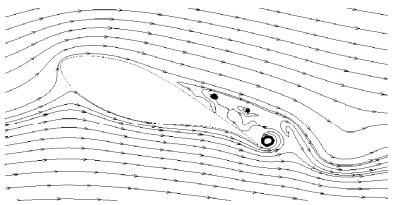

(d) $\alpha=22.91^{\circ}$ ( 上仰)

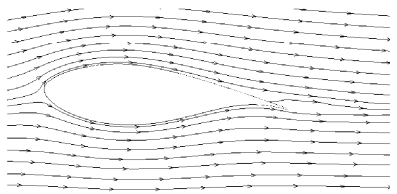

(f) $\alpha=4.97^{\circ}$ (下俯 )
图 6 动态失速典型时刻光滑叶片段流线图
继续上仰, 升阻力系数开始降低, 到 $D$ 点为局部最 小值，此后又开始增大，形成波动。如图 6c、6d 显示, 随着分离区域加大, 叶片段尾缘处不同尺度 的分离浴脱落的现象更加明显, 所以导致升阻力系 数波动加剧。光滑叶片段上仰至最大攻角后开始下 俯, 由于迟滞效应，在下俯阶段上翼面分离范围继 续扩大, 分离区流动变得复杂, 浴不断地从叶片段 后缘处脱落, 如点 $E$ (图 4、5)处, 此时攻角为 $20.25^{\circ}$ 。 随叶片段继续下俯, 流动分离现象开始缓和, 攻角 下俯至 $4.97^{\circ}$ 叶片段表面流动基本附着, 如图 6f 所示。

\subsubsection{VGs 对动态失速的影响}

图 7 与图 8 分别为攻角变化周期内加 VGs 前、后 叶片段升阻力系曲线的对比结果, 并选取动态失速过 程中 $B 、 D 、 E 、 G$ 四个时刻的升阻力系数进行分析, 此处 $B 、 D 、 E$ 点与图 4、5 对应时刻一致, $G$ 点为新 增时刻。如图 7 显示，上仰过程中，加 VGs 后叶片段 升力系数迟滞曲线环变小, 曲线光滑, 其线性部分变 宽, 在 $D$ 点处升力系数达到最大。在 $\alpha>15^{\circ}$ 后, 加 VGs 叶片段升力系数明显高于光滑叶片段。下俯过程 中, 加 VGs 叶片段升力系数在整个攻角范围内都明显 高于光滑叶片段, 在 $G$ 点后与上仰过程升力系数曲线 接近重合，迟滞性基本消失，迟滞现象得到改善。 图 8 显示了加 VGs 前、后的阻力系数变化曲线。如图 8 所示, 上仰过程中, 加 VGs 叶片段阻力系数在 $\alpha<15^{\circ}$ 范围内较光滑叶片无优异表现, 在 $\alpha>15^{\circ}$ 后则明显 低于光滑叶片段。下俯过程中, 整个攻角范围加 VGs 叶片段的阻力系数均大幅小于光滑叶片段。

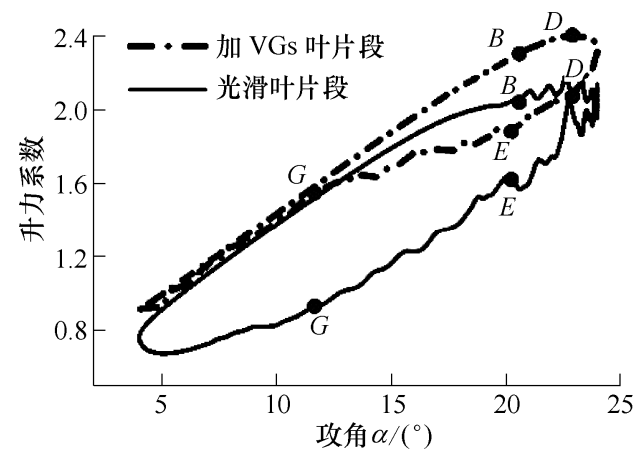

图 7 升力系数迟滞曲线对比

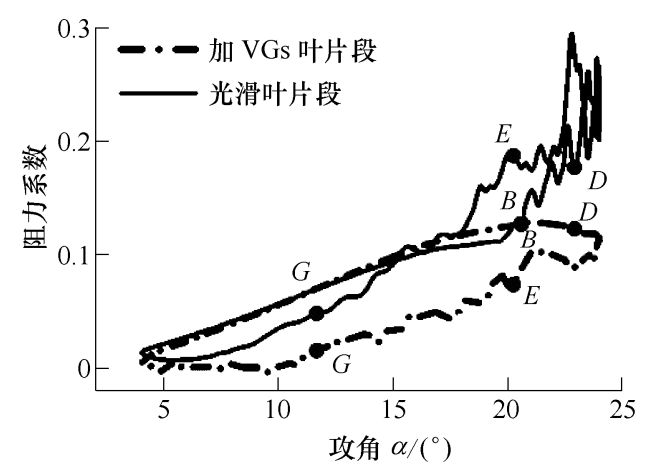

图 8 阻力系数迟滞曲线对比 
总体而言, 加 VGs 后, 叶片段最大升力系数增 幅为 $10 \%$, 周期内平均升力系数增幅为 $17.9 \%$; 最 大阻力系数减幅为 $56.3 \%$, 周期内平均阻力系数减 幅为 $40.3 \%$, 周期内平均升阻比增幅为 $97.5 \%$ 。

以上表明, VGs 在动态失速过程中具有显著增 升减阻的效果, 改善了气动力的迟滞现象, 安装 VGs 可提高动态失速下的风力机叶片气动性能。

为解释图 7 和图 8 中曲线变化的现象。图 9 给 出了动态失速过程 $B 、 D 、 E 、 G$ 四个时刻光滑叶片 段(左)与加 VGs 叶片段(右)展向中心剖面浴量云图。 如图 9 所示, 当叶片段上仰至 $B$ 点 $\left(\alpha=20.60^{\circ}\right)$ 时, 光滑叶片段上表面后缘处出现较为严重地分离, 加 VGs 叶片段上表面的边界层流动基本处于附着状 态, 并未出现分离现象, 这是由于 VGs 产生的高能 量浴流与下游低能量边界层流体混合, 从而增强了 叶片段表面流体抵抗逆压梯度的能力; 在 $D$ 点 $\left(\alpha=22.91^{\circ}\right)$ 时, 光滑叶片段表面分离较为严重, 分离 点靠近前缘, 分离浴脱落形成尾浴; 加 VGs 叶片段 上表面尾缘处仅发生了轻微地分离, 流体仍然附着 在叶片表面, 升力系数达到最大。最大攻角后开始 下俯, 从图 $9 \mathrm{c}$ 可以看出, 动态失速对下附过程中分 离流的闭合有延迟作用, 导致在低攻角 $E$ 点相比高 攻角 $D$ 点的分离现象更加严重, 脱落涡结构更明显; 加 VGs 后, VGs 将光滑叶片的外侧的高能量流体席
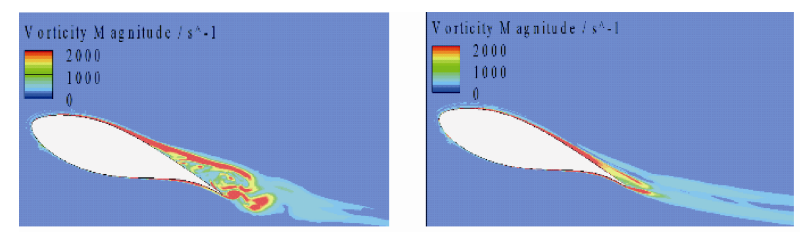

(a) $B$ 点, $\alpha=20.60^{\circ}$ ( 上仰, 左: 光滑, 右: 加 VGs)
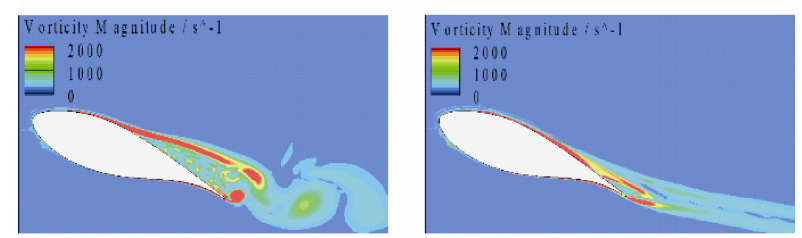

(b) $D$ 点, $\alpha=22.91^{\circ}$ ( 上仰, 左: 光滑, 右: 加 VGs)
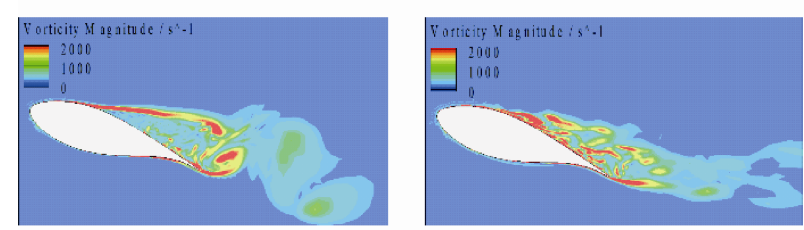

(c) $E$ 点, $\alpha=20.25^{\circ}$ (下俯, 左: 光滑, 右: 加 VGs)
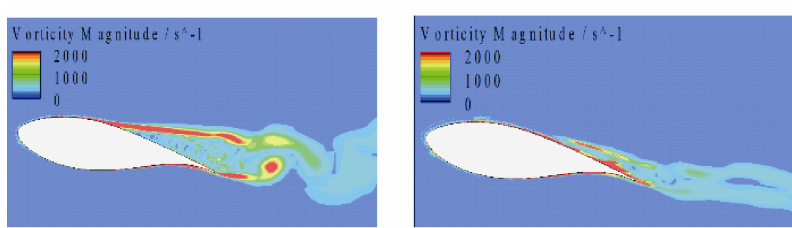

(d) $G$ 点, $\alpha=11.66^{\circ}$ (下俯, 左: 光滑, 右: 加 VGs)

图 9 过程点有无 VGs 叶片段涡量云图
卷到近壁面, 如图 9c 所示, 在低能量和高能量流体 汇集过程中, 叶片表面聚集了高浴量的流体，并形 成小尺度浴。下俯至 $G$ 点 $\left(\alpha=11.66^{\circ}\right)$ 时, 光滑叶片 段上表面分离依然非常剧烈, 加 VGs 叶片段流动则 已呈附着现象。

以上表明, 动态失速下, 光滑叶片段上仰时的 分离与下俯时的重新附着均出现延迟, 加 VGs 后发 现, 上仰阶段叶片段的气动分离更加延迟, 下俯阶 段叶片段上表面附着流动重建的更早。

\section{3 模拟方法对 VGs 抑制作用预测影响分析}

图 10、11 分别为 $k-\omega$ SST 模型与 DDES 方法得 到的加 VGs 叶片段升、阻力系数迟滞曲线。从升、 阻力系数曲线均可看出, 叶片段上仰阶段, 两模拟 方法计算结果在 $\alpha<20^{\circ}$ 攻角下吻合良好, 但在 $\alpha>20^{\circ}$ 大攻角下偏离明显。这是因为, 在低攻角下叶片段 表面流动整体为附着流动, 涡结构较小, 基于 $k-\omega$ SST 模型的 RANS 模拟方法可以准确模拟附着流 动, 故与 DDES 模拟结果一致。当叶片段上仰至大攻 角产生严重流动分离时, DDES 与 $k-\omega \mathrm{SST}$ 模拟结果 差异明显, $k-\omega \mathrm{SST}$ 模拟值偏高, 这是因为 $k-\omega \mathrm{SST}$ 不能准确预测叶片分离点的位置和捕捉叶片分离浴 结构。下俯阶段, 叶片段表面分离流动复杂, 两模拟 方法的升阻力系数结果差异更加明显, 表现为 $k-\omega$ SST 的升力系数模拟值高于 DDES 模拟值, 其阻力系 数则低于 DDES 模拟值。形成差异的原因为 DDES 方 法在分离区域可以更细致地捕捉到涡结构信息。

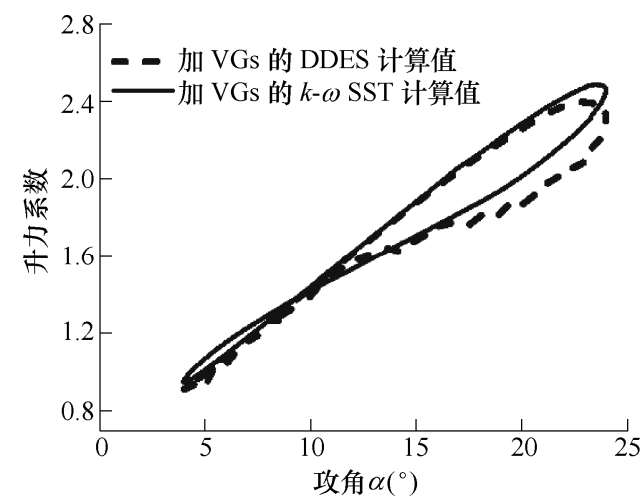

图 10 两种模拟方法升力系数迟滞曲线对比

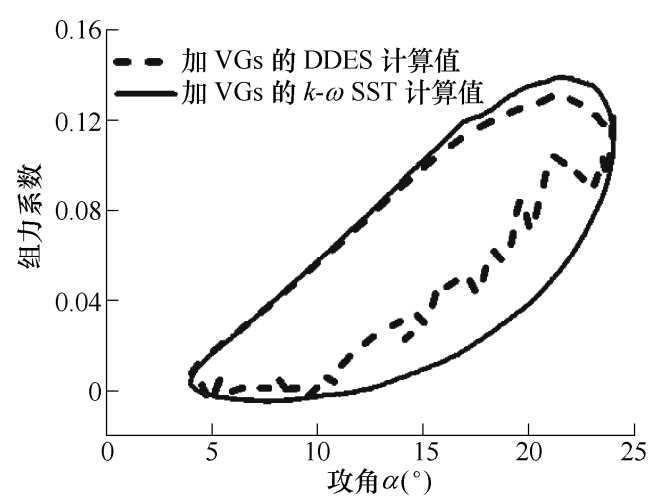

图 11 两种模拟方法阻力系数迟滞曲线对比 
图 12 给出了下俯至 $\alpha=21.85^{\circ}$ 时加 VGs 叶片段 展向中心剖面的流线图, 图 $12 \mathrm{a}$ 为 DDES 模拟结果, 图 $12 \mathrm{~b}$ 为 $k-\omega \mathrm{SST}$ 模拟结果。从图 $12 \mathrm{a}$ 明显可以看 出, DDES 模拟结果反映了许多小尺度浴结构, 流 场形态复杂, 更加接近于实际; $k-\omega \mathrm{SST}$ 结果中仅 反映了一个尾缘处的大尺度分离浴, 流场形态简单。 这是由于 DDES 模型具有 LES 模型对小尺度浴结构 更好的求解能力的优势, 而 RANS 方法因时均化后 对流场速度脉动求解不够, 仅能够捕捉大尺度浴结 构, 难以预测到更细小的流场细节。因此, 相对于 流动复杂、分离浴结构不断复杂变化的动态失速而 言, DDES 模拟方法因可捕捉到流场中小尺度浴的 产生、发展、脱落的变化过程, 而更加适合。

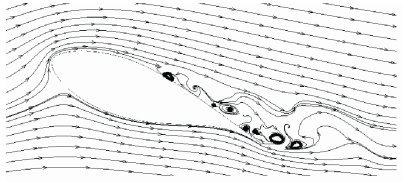

(a) DDES

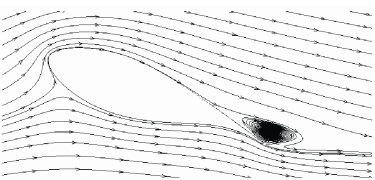

(b) $k-\omega \mathrm{SST}$
图 12 加 VGs 叶片段流线图， $\alpha=21.85^{\circ}$ (下俯)

如图 13 所示, 两模拟方法得到 VGs 对浴量影响 的预测也有较大差异, 对 VGs 作用下形成的高、底能 量流体混合过程预测产生更大差异, 显然 DDES 得到 的浴量不均匀分布的涡结构更加合理, 对于解释 VGs 对流动的控制机理更加有益。二维光滑叶片相关研究 指出 RANS 方法难以体现湍流的三维特性。从图 14 可看出, 尽管加 VGs 后叶片表面流动的三维强湍流特 性加剧, $k-\omega \mathrm{SST}$ 计算结果沿展向仍体现了近二维性 的脱落浴结构; DDES 模型则得到复杂的展向与流向 相互扰动的脱落浴结构, 具有明显的三维性。由此说 明, $k-\omega \mathrm{SST}$ 模型对加 VGs 叶片段动态失速下分离产 生的复杂湍流三维性的模拟效果很差。

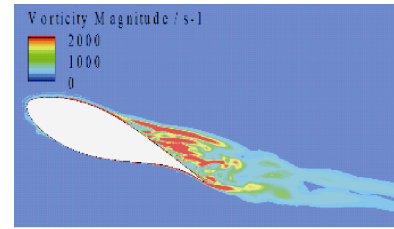

(a) DDES

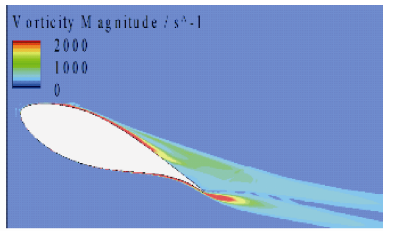

(b) $k-\omega \mathrm{SST}$
图 13 加 VGs 叶片段浴量云图, $\alpha=23.85^{\circ}$ (下俯)

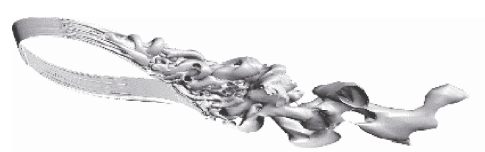

(a) DDES

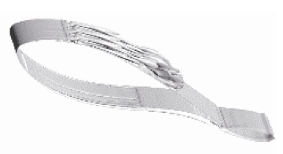

(b) $k-\omega \mathrm{SST}$
图 14 加 VGs 叶片段 $\mathrm{Q}$ 判据为 0.001 的浴量 等值面图, $\alpha=21.85^{\circ}$ (下俯)

\section{3 结论}

采用基于 $k-\omega$ SST 的 RANS 方法和 DDES 模型,
对 DU91-W2-250 叶片段加 VGs 前后的动态失速过 程进行了模拟计算, 结果如下。

(1) 静态失速下, DDES 对升力系数模拟结果与 试验值吻合良好; $k-\omega$ SST 模拟方法在失速之前精 度较高, 在静态失速区域 $k-\omega \mathrm{SST}$ 模型未能很好预 测分离现象，预测精度低于 DDES 方法。

(2) 动态失速下, 光滑叶片段上仰时的分离与 下俯时的重新附着均出现延迟, 气动力出现严重的 迟滞现象, 加 VGs 后, 上仰阶段叶片段的气动分离 更加延迟，下俯阶段叶片段上表面附着流动重建的 更早，气动力的迟滞现象得到明显改善。

(3) 动态失速下, VGs 具有明显的增升减阻效 应。在 DU91-W2-250 叶片段上仰过程中, 最大升力 系数增加 $10 \%$ ，周期内平均升力系数增加 $17.9 \%$; 最大阻力系数减少 $56.3 \%$, 周期内平均阻力系数减 少 $40.3 \%$ ；周期内平均升阻比增加 $97.5 \%$ 。

（4）基于 RANS 方法的 $k-\omega$ SST 模型对动态失 速过程中分离涡细节捕捉严重不足，对动态失速下不 同尺度浴结构反映不够清晰。DDES 模拟方法更适合 用于分析动态失速等问题的复杂分离流动现象。

\section{参 考 文 献}

[1] 李涛, 赵振宙, 陈景茹, 等. 考虑转捩的风力机浴流发 生器数值模拟 [J]. 机械工程学报, 2017, 53(4): 149-154. LI Tao, ZHAO Zhenzhou, Chen Jingru, et al. Numerical simulation of the wind turbine vortex generators with transition modeling $[\mathrm{J}]$. Journal of Mechanical Engineering, 2017, 53(4): 149-154.

[2] 赵振宙, 严畅, 王同光, 等. 考虑叶片相互影响风力机 浴流发生器参数化建模 $[\mathrm{J}]$. 机械工程学报, 2018, 54(2): 201-208.

ZHAO Zhenzhou, YAN Chang, WANG Tongguang, et al. Parametric model of vortex generators of wind turbine considering inter-effect of winglets[J]. Journal of Mechanical Engineering, 2018，54(2): 201-208.

[3] TIMMER W A, VANROOY R P J O M. Summary of the Delft university wind turbine dedicated airfoils[J]. Journal of Solar Energy Engineering, 2003， 125(4): 488-496.

[4] TIMMER W A, VANROOY R P J O M. Wind tunnel results for a $25 \%$ thick wind turbine blade airfoil[C]//European Community Wind Energy Conference, March 8-12, 1993, Lubeck-Travemunde, Gemany, 1993: 416-419.

[5] ZHANG Lei, YANG Ke, XU Jianzhong. Effects on wind turbine airfoils by vortex generators[J]. Journal of Engineering Thermophysics, 2010, 31(5): 749-752.

[6] MANOLESOS M, VOUTSINAS S G. Experimental investigation of the flow past passive vortex generators on 
an airfoil experiencing three-dimensional separation[J]. Journal of Wind Engineering and Industrial Aerodynamics, 2015, 142: 130-148.

[7] ZHANG Lei, LI Xingxing, YANG Ke, et al. Effects of vortex generators on aerodynamic performance of thick wind turbine airfoils $[\mathrm{J}]$. Journal of Wind Engineering and Industrial Aerodynamics, 2016, 156: 84-92.

[8] GAO Linyue, ZHANG Hui, LIU Yongqian, et al. Effects of vortex generators on a blunt trailing-edge airfoil for wind turbines[J]. Renewable Energy, 2015, 76: 303-311.

[9] 何政洋. 浴流发生器参数对风力机叶片气动特性影响 的数值模拟研究 [D]. 重庆: 重庆大学, 2016.

HE Zhengyang. Numerical simulation investigation of effect of vortex generator parameters on aerodynamic characteristics of wind turbine blade[D]. Chongqing: Chongqing University, 2016.

[10] 郝礼书, 乔志德, 宋文萍. 浴流发生器布局方式对翼型 失速流动控制效果影响的实验研究 $[\mathrm{J}]$. 西北工业大学 学报, 2011, 29(4): 525-528.

HAO Lishu, QIAO Zhide, SONG Wenping. Experimentally studying effects of different layouts of vortex generator on controlling stall flow over airfoil[J]. Journal of Northwestern Polytechnical University, 2011, 29(4): 525-528.

[11] MELIUS M, CAL R B, MULLENERS K. Dynamic stall of an experimental wing turbine blade[J]. Physics of Fluids, 2016, 28(3): 034103.

[12] 胡丹梅, 李佳, 间海津. 水平轴风力机翼型动态失速的 数值模拟 $[\mathrm{J}]$. 中国电机工程学报, $2010,30(20)$ : 106-111.

HU Danmei, LI Jia, YAN Haijin. Numerical simulation of airfoil dynamic stall of horizontal axis wind turbine[J]. Proceedings of the CSEE, 2010，30(20): 106-111.

[13] LIU Xiong, LU Cheng, GODBOLE At, et.al. Effects of aerodynamic damping on the tower load of offshore horizontal axis wind turbines[C]//8th International Conference on Applied Energy, Abu Dhabi, U ARAB EMIRATES: APPLIED ENERGY, 2017, 204: 1101-1114.

[14] LIU Xiong, LU Cheng, GODBOLE A, et al. Vibration-induced aerodynamic loads on large horizontal axis wind turbine blades[C]//7th International Conference on Applied Energy, Abu Dhabi, U ARAB EMIRATES: APPLIED ENERGY，2017，185: 1109-1119.

[15] 李德源, 汪显能, 莫文威, 等. 动态气动载荷和构建振 动对风力机气弹特性的影响分析 [J]. 机械工程学报, 2016, 52(14): 165-173.

LI Deyuan, WANG Xianneng, MO Wenwei, et al. Analysis on the influence of dynamic aerodynamic loads and component vibration of wind turbine on aeroelastic characteristics[J]. Journal of Mechanical Engineering, 2016, 52(14): 165-173.

[16] BENTON S I, VISBAL M R. The onset of dynamic stall at a high transitional Reynolds number[J]. Journal of fluid Mechanics, 2018, 861: 860-855.

[17] 戴丽萍, 许雅苹, 周强, 等. 浴流发生器对风力机翼型 动态失速的影响 $[\mathrm{J}]$. 工程热物理学报, 2018, 39(8): 1707-1712.

DAI Liping, XU Yaping, ZHOU Qiang, et al. Numerical simulation of pitching wind turbine airfoil dynamic stall[J]. Journal of Engineering Thermophysics, 2018, 39(8): 1707-1712.

[18] 王圣业, 王光学, 董义道, 等. 基于雷诺应力模型的高 精度分离涡模拟方法 [J]. 物理学报, 2017，66(18)： 187401.

WANG Shengye, WANG Guangxue, DONG Yidao, et al. High-order detache3d-eddy simulation method based on a Reynolds-stress background mode[J]. Acta Physics Sinica, 2017, 66(18): 187401.

[19] MORTON S A, STEENMAN M, et al. Multidisciplinary applications of detached-eddy simulation to separated flows at high Reynolds numbers[C]//IEEE Computer Society. Users Group 2003 Conference, Proceedings, Bellevue, WA, IEEE Computer Society, 2003: 113-128.

[20] 梁志成. 用分离浴方法对梢浴流动的数值模拟 [D]. 上 海：上海交通大学，2012.

LIANG Zhicheng. Detached eddy simulation of wing tip vortex[D]. Shanghai: Shanghai Jiao Tong University, 2012.

[21] SPALART P R, DECK S, SHUR M L, et al. A new version of detached-eddy simulation, resistant to ambiguous grid densities[J]. Theoretical and Computational Fluid Dynamic, 2006, 2: 181-195.

[22] LETTGUS J, GARDNER A D, SCHWERMER T. Numerical investigations of dynamic stall on a rotor with cyclic pitch control[J]. Journal of the American Helicopter Society, 2019, 64(1): 1-14.

[23] 龚杰, 郭春雨, 吴铁成, 等. 与基于分离浴模拟方法的 导管桨近尾流场及尾浴特性分析 [J]. 上海交通大学学 报，2018，52(6): 674-680.

GONG Jie, GUO Chunyu, WU Tiecheng, et al. Detached eddy simulation of near wake field and vortex characteristics for a ducted propeller[J]. Journal of Shanghai Jiao Tong University, 2018, 52(6): 674-680.

作者简介: 赵振宙(通信作者), 男, 1982 年出生, 博士后, 教授。主要 研究方向为风力机气动流场控制。

E-mail: joephy@163.com 